\title{
Role of H. pylori Infection (Serology, PCR) in Chronic Idiopathic Thrombocytopenic Purpura in an Endemic Country: A Case Control Study, Tehran, IRAN
}

\section{Mohammad Faranoush ${ }^{1}$, Samileh Noorbakhsh ${ }^{2},{ }^{*}$, Azim Mehrvar ${ }^{3}$ and zardokht Tabatabae}

${ }^{1}$ Associate Professor in Pediatric Hematology \& Oncology, Research Center of Pediatric Infectious Diseases Tehran University of Medical Sciences Tehran, Iran ${ }^{2}$ Full Professor in Pediatric Infectious Disease, Research Center of Pediatric Infectious Diseases Tehran University of Medical Sciences Tehran, Iran

${ }^{3}$ Assistant Professor in Pediatric Hematology \& Oncology, Research Center of Pediatric Infectious Diseases Tehran University of Medical Sciences Tehran, Iran ${ }^{4}$ Microbiology, Research Center of Pediatric Infectious Diseases Tehran University of Medical Sciences, Tehran, Iran

\begin{abstract}
Background: A practical guideline for detection and managements of some common infectious agents in cases with chronic ITP (Idiopathic Thrombocytopenic Purpura) is so important.

Objectives: to investigate the role of $H$. pylori infection in children with chronic ITP in an endemic area.

Materials and methods: A case control study done in pediatric ward Rasul Hospital, Tehran, Iran (2009-2010). 51 chronic ITP cases and 25 controls were assessed. H. pylori IgG \& IgA ELISA (LDN -Germany) assesses in all cases and controls. All cases undergoing Bone Marrow Aspiration. H. pylori-PCR evaluated (QIAquickP® QIAGEN; Germany). P-value $<0.05$ was considered statistically significant.

Results: cases were between $1-20$ years (mean 13.3.5 \pm 7.6 y). Platelet count varied between $5000-1330000$ (mean $63621 \pm 37369.9)$ Positive $H$. pylori- IgA observed in $70 \%(36 / 51)$ of cases and $4 \%(1 / 25)$ of controls: $\mathrm{p}$-value $=0.00$. H. pylori $(\mathrm{IgG})$ was not significantly difference between cases and controls. $[51 \%(26 / 51)$ vs. $32 \%$ $(8 / 25)$, p-value $=0.09$ ] .Poor agreement observed between $H$. pylori $-\lg A$ and $H$. pylori $-\lg G$ antibodies and severity of thrombocytopenia in ITP cases (Kappa=-0.11; 0.04). Positive PCR results was \% 5.9\% (3/51) in ITP cases without significant difference in age between positive and negative PCR results (mean age $9.3 \pm 9.7$ years vs. $13.5 \pm 7.52$ years; $p$-value $=0.3$ ) Poor agreement between positive PCR and positivity of IgA (actual agreement=47.062\%; $p$-value $=0.5 ; \mathrm{Kappa}=-0.04$ ), and IgG antibodies (actual agreement $=40.91 \% ; \mathrm{p}$-value $=0.6 ; \mathrm{Kappa}=-0.04$ respectively) were observed in ITP cases.

Conclusion: We concluded that $H$. pylori infection (serologically) is high in young Iranian population. In chronic ITP, the H. pylori infection can be considered as an additional disorder which aggravates the main disease. The management of mild-to-moderate chronic ITP in Iranian patients, especially those with a recent onset of disease, should include an investigation for and eradication of infection with $\mathrm{H}$. pylori.
\end{abstract}

Keywords: H. pylori; ITP (Idiopathic Thrombocytopenic Purpura); H. pylori IgG; IgA; PCR

\section{Introduction}

Idiopathic Thrombocytopenic Purpura (ITP) is defined as a characteristic rash associated with an abnormally low platelet count of unknown cause. In opinion of James and other authors Idiopathic Thrombocytopenic Purpura (ITP) defined as isolated thrombocytopenia with no clinically apparent associated conditions or other causes of thrombocytopenia. Exclusion of recognized alternative etiologies of thrombocytopenia was the basis for the idiopathic thrombocytopenic Purpura [1]. HIV; hepatitis C infections and H. pylori infection should be considered an alternative disorder [1-5].

Scandellari et al. showed a cross-reaction of an H. pylori urease B monoclonal antibody with platelet glycoprotein IIIa and suggested that the immune response to UreB may be involved in the pathogenesis of ITP [2]. The possible role of $H$. pylori infection in the development of ITP had studied in some systemic reviews [3-5]. Arnold et al. showed an overall platelet response in more than $50 \%$ of the patients successfully treated for the infection and increased response rates in countries with a high prevalence of $H$. pylori infection in background populations, i.e. in patients with less severe degrees of thrombocytopenia and in those with shorter disease duration. [1]

Figura et al. [5] reported that the cure of $H$. pylori infection totally corrects the thrombocytopenia in certain patients. In other patients with ITP, the infection can be considered as an additional disorder which aggravates the main disease, while in a third group of patients the eradication of $H$. pylori appears to have no effect on the course of thrombocytopenia [5]. H. pylori is a gram negative bacterium and considered the etiologic agent of some gastrointestinal and extra gastrointestinal as a class I carcinogen by the World Health Organization. Colonization of $\mathrm{H}$. pylori has been found in dental plaques, saliva, tonsils, and sinus mucosa. $H$. pylori infection varies among countries and often within a country [6]. ITP in adult patients may be associated with serum antibodies. There are geographical disparities of both the frequency of $H$. pylori infection among patients with ITP and the frequency of platelet count responses following eradication of $H$. pylori infection, and these two frequencies correlate with each other [1]. There

*Corresponding author: Samileh Noorbakhsh, Research Center of Pediatric Infectious Diseases, $4^{\text {th }}$ floor Rasoul Hospital, Niayesh Street, Satarkhan Avenue, Tehran, 14455 Islamic Republic of Iran, Tel: 00982166525328; Fax: 00982166516049; E-mail: Samileh_noorbakhsh@yahoo.com, noorbakhgsh@tums.ac.ir

Received March 11, 2013; Accepted May 10, 2013; Published May 18, 2013

Citation: Faranoush M, Noorbakhsh S, Mehrvar A, Tabatabae Z (2013) Role of $H$. pylori Infection (Serology, PCR) in Chronic Idiopathic Thrombocytopenic Purpura in an Endemic Country: A Case Control Study, Tehran, IRAN. J AIDS Clin Res 4: 209. doi:10.4172/2155-6113.1000209

Copyright: (c) 2013 Faranoush M, et al. This is an open-access article distributed under the terms of the Creative Commons Attribution License, which permits unrestricted use, distribution, and reproduction in any medium, provided the original author and source are credited. 
exist an inconsistency among previous studies which has prevented broad acceptance of the association of $\mathrm{H}$. pylori infection with ITP. Moreover, the etiologic factors of ITP in the Iranian population are not well understood [7-12]. The etiologic factors of ITP in Iranian population are not well understood. H. pylori infection in the Iranian population is high [13-18]. Positive serum $H$. pylori- IgA reported in $15 \%$ and positive $H$. pylori - IgG in $11 \%$ of children with rhino sinusitis $>2$ weeks (mean age 4.2 years)

Except 1 study in Iranian adults, the relationship between $H$. pylori infection and ITP was not explained until yet. Rostami et al. reported the eradication of $\mathbf{H}$. pylori on platelet recovery in patients with chronic ITP [19]. Providing the practical guidelines for detection and managements of some common infectious organisms like as $H$. pylori infection in ITP cases is so important in our country.

This case control study in children in Iran was conducted in order to investigate the role of $H$. pylori infection in cases with chronic ITP.

\section{Material and Methods}

A cross sectional study performed in the Department of pediatrics, Rasul Hospital, Tehran, Iran (2009 - 2010) The study was approved by the ethical Committee in Research Center of Pediatric Infectious Disease in Tehran University of Medical Sciences. We studied 51 consecutive Iranian patients with chronic ITP and 25 normal controls.

Initially a questionnaire was completed by an authorized physician, followed by a complete clinical examination. All cases and controls were examined by an internist for other concomitant disorders (immune deficiencies state; diabetes mellitus, renal/ heart failure; etc).Blood samples were taken for routine blood tests as well as serologic tests before BMA. Blood samples $(2 \mathrm{ml})$ were centrifuged and transferred to our research laboratory. The serum was stored at $-20^{\circ} \mathrm{C}$ until the serologic examination was performed. Specific $H$. pylori antibodies ( $\operatorname{IgG} \& \operatorname{IgA}$ ) in all cases and controls were assessed by ELISA. The commercial kits (Chemicon-Germany) were used and the results were interpreted as suggested by the manufacturer. Results were calculated quantitatively.

Bone Marrow Aspiration (BMA) had done in all studied cases. BMA samples placed in sterile tubes. Samples were centrifuged and homogenized, then preserved in $-80^{\circ} \mathrm{C}$. A PCR template Purification Kit (Roche; Germany) was used. The binding column tube was transferred to a new $1.5 \mathrm{ml}$ tube. The integrity of DNA was assessed by gel electrophoresis ( $1 \%$ agarose). $H$. pylori- DNA was evaluated qualitatively by specific PCR primer kits (QIA quick P $^{\circledast}$ QIAGEN; Germany). Diagnostic kits included a ready to use PCR mix Kit, positive and negative controls and other qualified reagents along with a protocol for detecting as low as 10 copies $/ \mathrm{ml}$ of the $H$. pylori genome.

\section{Statistical analysis}

The student's $t$ test was used to determine significant differences in means for continuous variables and chi-square for comparing categorical data in cases and controls. P-value s less than 0.05 were considered statistically significant.

The agreement between serologic test and PCR was assessed by the calculation of Kappa statistic. Landis and Koch suggested that a kappa greater than 0.75 represents excellent agreement beyond chance, a kappa below 0.40 represents poor agreement, and a Kappa of 0.40 to 0.75 represents intermediate to good agreement.

\section{Results}

\section{Demographic results}

$45 \%$ (23) of cases were male and 55\% (28) female. Ages varied between 1 to 20 years; mean $13.3 .5 \pm 7.6$ years (Figure 1$) .37 .3 \%(19 / 51)$ of studies cases was young $(<10$ years $)$ and $63 \%(32 / 51)$ was old $(>10$ years).

Mean age of ITP cases in males (11.6 \pm 8 years) was insignificant ( $\mathrm{p}$-value $=0.1$ ) with female $(14.6 \pm 7.2$ years). Platelet count varied between 5000-1330000; mean $63621 \pm 37369$.9. Platelet count was not different between male $(62365 \pm 40000)$ and female $164653 \pm 35741)$ $\mathrm{p}$-value $=0.8$.

Sever ITP $($ PLT $<20 \times 109 / \mathrm{L}$ 0000) detected in $11.6 \%(6 / 51)$; Moderate ITP $(\mathrm{PLT}<20-80 \times 109 / \mathrm{L} 000)$ in $54.9 \%(28 / 51)$; and Mild ITP $(>80 \times 109 / \mathrm{L})$ in $33.3 \%(17 / 51)$ of cases. Serologic results: Positive H. pylori- IgA observed in $70 \%(36 / 51)$ of cases and $4 \%(1 / 25)$ of controls. Serum level for $H$. pylori- IgA in ITP cases was between 0.2-85 mg percent, mean $14 \pm 18$.

The mean level of H.pylori-IgA in female cases was higher than male cases. $(18.4 \pm 21.9$ vs. $8.7 \pm 10 \mathrm{mg} \%$; p -value=.055). H.pyloriIgA positively was significantly higher in cases. [70\% vs. $4 \%$ of controls, p-value $=0.00]$. Serum H.pylori- IgG level was between 0.2-119 mg\%; mean $17.2 \pm 25$.3. The mean level of H.pylori- $\operatorname{IgG}$ had not significant differences between male and female $(15.2 \pm 30$ vs. $18.8 \pm 19.9 \mathrm{mg} \%$; $\mathrm{p}$-value $=0.6)$. H. pylori $(\mathrm{IgG})$ was not significantly difference between cases and controls [51\% (26/51) vs. $32 \%(8 / 25)$, p -value=0.09]. Poor agreement observed between $H$. pylori - IgA and $H$. pylori - IgG antibodies with severe ITP (Kappa=-0.11; 0.04) (Tables 1 and 2).

\section{PCR results}

Positive H. pylori-PCR in BMA was $\% 5.9 \%$ (3/51. The mean age was not different between ITP cases with positive and negative PCR results (mean age $9.3 \pm 9.7$ years vs. $13.5 \pm 7.52$ years; $p$-value $=0.3$ ).

Poor agreement observed between positive PCR IgA (actual agreement $=47.062 \%$; -value $=0.5$; Kappa $=-0.04)$; and $\operatorname{IgG}$ antibodies (actual agreement $=40.91 \% ; \mathrm{p}$-value $=0.6 ; \mathrm{Kappa}=-0.04$, respectively) (Tables 3-5).

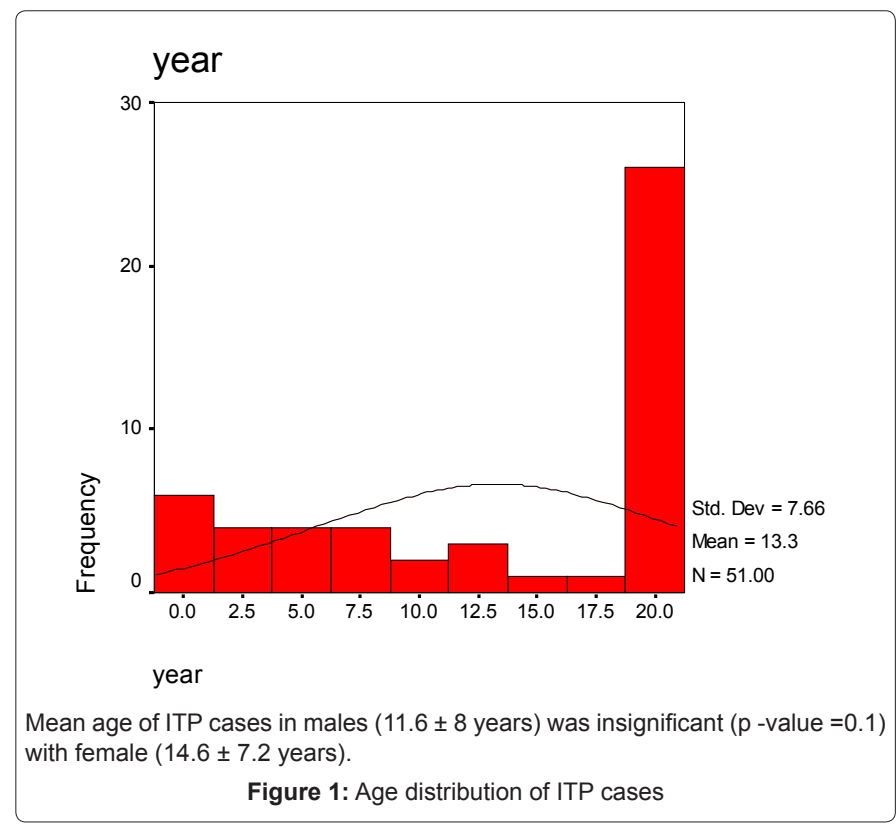




\begin{tabular}{|l|l|l|l|l|}
\hline \multicolumn{3}{|l|}{ Observer 2 } & & \\
\hline & + & - & & \\
\hline+ & 14 & 8 & 22 & $73.33 \%$ \\
\hline- & 6 & 2 & 8 & $26.67 \%$ \\
\hline & 20 & 10 & 30 & \\
\hline & Actual agreement= & $53.33 \%$ & \\
\hline & $\begin{array}{l}\text { Chance agreement }= \\
\text { Kappa statistic= }\end{array}$ & $57.78 \%$ & \\
\hline
\end{tabular}

Table 1: Correlation between positive IgA and severe ITP (PLT count <80000).

\begin{tabular}{|l|l|l|l|l|l|}
\hline & \multicolumn{3}{|l|}{ Observer 2 } & & \\
\hline \multirow{2}{*}{ Observer 1 } & + & 9 & 4 & 13 & $43.33 \%$ \\
\cline { 2 - 5 } & - & 11 & 6 & 17 & $56.67 \%$ \\
\hline & & 20 & 10 & 30 & \\
\hline & \multirow{2}{*}{ Actual agreement= } & $50.00 \%$ & \\
\hline & \multirow{2}{*}{ Chance agreement= } & $47.78 \%$ & \\
\hline & \multirow{2}{*}{ Kappa statistic= } & $\mathbf{0 . 0 4}$ & \\
\hline
\end{tabular}

Table 2: Correlation between positive IgG and sever ITP.

\begin{tabular}{|l|l|l|l|l|l|}
\hline & \multicolumn{3}{|l|}{ Observer 2 } & & \\
\hline \multirow{2}{*}{ Observer 1 } & + & 3 & 23 & 26 & $59.09 \%$ \\
\hline & - & 3 & 15 & 18 & $40.91 \%$ \\
\hline & 6 & 38 & 44 & \\
\hline & & Actual agreement= & $40.91 \%$ & \\
\hline & Chance agreement= & $43.39 \%$ & \\
\hline & & Kappa statistic= & $-\mathbf{0 . 0 4}$ & \\
\hline
\end{tabular}

Table 3: Agreement between positive PCR IgA and IgG antibody.

Poor agreement (actual agreement $=37.2 \%$; p-value $=0.6$; Kappa $=-0.06$ ) detected between positive $H$. pylori $-\mathrm{PCR}$ and severity of ITP (PLT count <80000) (Table 6).

\section{Discussion}

We studied 51 chronic ITP cases aged between 1 to 20 years (mean13.3.5 \pm 7.6 years), $63 \%$ of cases was older than 10 years. $54.9 \%$ of cases had Moderate thrombocytopenia (20000-80000), severe thrombocytopenia $(\mathrm{PLT}<200000)$ detected only in $11.6 \%$.

H. pylori -DNA was positive in Bone marrow aspiration of $5.9 \%$ $(3 / 51)$ cases with mean age 10 years, without difference in mean age of cases with positive and negative results ( $\mathrm{p}$-value $=0.3) .70 \%(36 / 51)$ of cases had Positive serum H.pylori- IgA in compare with $4 \%(1 / 25)$ of controls ( $\mathrm{p}$-value $=0.00)$.

Positive serum H. pylori -IgG observed in $51 \%$ of cases in compare with $32 \%$ in controls without significant difference ( $\mathrm{p}$-value $=0.09$ ). Severity of thrombocytopenia in ITP cases had poor agreement with positive $H$. pylori $-\operatorname{IgA}$ (Kappa index=-0.11); positive $H$. pylori - IgG antibodies (Kappa index $=0.04)$; and positive H. pylori $-\mathrm{DNA}(\mathrm{PCR})$ in BMA (Kappa index $=-0.06$ ). Positive $H$. pylori-DNA obtained in BMA of $5.9 \%$ ITP cases (mean age $=10$ years) was less frequent than 3 previous Iranian studies.

Chronic and persistent infection (positive-PCR) might be found in parts of upper respiratory tract (nasal polyp, adenoid tissues) for longer period. H. pylori infection was detectable in adenoid tissues of $15 \%$ children before 8 years which is lower than Khadem et al. study on adults cases [14-16]. Saffari et al. studied $H$. pylori antibodies in population in Shiraz (south of Iran) [14]. Seroprevalence to H. pylori infection is high in Iranian population [14-18]. Initial infection probably occurs at an early age, its prevalence increases with age. The infection will increase to $30 \%$ in 2 nd and $53.5 \%$ after $4^{\text {th }}$ decade of life [16]. $28.3 \%$ of persons between $20-40$ years; $32 \%$ of population between 41-80 years had positive $H$. pylori-IgG. But recent $H$. pylori infection (positive IgA) observed in $4 \%$ of controls are lower than previous studies $(16.7 \%, 53.5 \%$ respectively).Higher age of cases might explain these differences. [17] Here, we found previous HP Infection (Positive IgG $=32 \%$ ) in controls which is very close to previous studies in normal Iranian population [14-18]. Although $H$. pylori infection varies between countries and often within a country, higher age for cases in Farhadi et al. study is the probable cause for this higher infection in compare with studied children [20].

In countries such as Japan and Italy, where most studies of $H$. pylori eradication in ITP have been performed, testing for $H$. pylori infection has been recommended. HP eradication was successful in $87 \%(62 / 71)$ of adult cases with ITP who completed the eradication [5]. In proven cases eradication therapy is recommended as the initial treatment in $H$. pylori-infected patients [5,6]. Rostami et al. study [19] defined $48 \%$ (30/62) of HP-eradicated patients showed an ITP response, none $(0 \%)$ of HP-negative ITP patient had improved. The ITP response persisted for 48 weeks in 93\% (28/30) of the responders. The ITP responders had a shorter disease duration than none responders, $p$-value $=0.002$ [19].

\section{Conclusion}

We concluded that $H$. pylori infection (serologically) is high in young Iranian population. In chronic ITP, the $H$. pylori infection can be considered as an additional disorder which aggravates the main disease. The management of mild-to-moderate chronic ITP in Iranian patients, especially those with a recent onset of disease, should include an investigation for and eradication of infection with $\mathrm{H}$. pylori.

\section{Acknowledgments}

This study was supported by the Research Center of Pediatric Infectious Diseases. We thank "Research Center of Cellular and Molecular Biology" at the Tehran University of Medical Sciences.

\begin{tabular}{|l|l|l|l|l|l|}
\hline & \multicolumn{3}{|l|}{ Observer 2 } & & \\
\hline \multirow{2}{*}{ Observer 1 } & + & - & & \\
\hline & + & 1 & 25 & 26 & $50.98 \%$ \\
\hline & & 3 & 23 & 25 & $49.02 \%$ \\
\hline & & 48 & 51 & \\
\hline & & Actual agreement= & $47.06 \%$ & \\
\hline & Chance agreement= & $49.13 \%$ & \\
\hline & & Kappa statistic= & $-\mathbf{0 . 0 4}$ & \\
\hline
\end{tabular}

Table 4: Agreement between positive PCR and IgG antibody.

\begin{tabular}{|l|l|l|l|l|l|}
\hline & \multicolumn{3}{|l|}{ Observer 2 } & & \\
\hline \multirow{2}{*}{ Observer 1 } & + & 3 & - & & \\
\cline { 2 - 6 } & - & 32 & 16 & 3 & $5.88 \%$ \\
\hline & & 35 & 16 & 51 & $94.12 \%$ \\
\hline & & Actual agreement= & $37.25 \%$ & \\
\hline & & Chance agreement= & $33.56 \%$ & \\
\hline & & Kappa statistic= & $\mathbf{0 . 0 6}$ & \\
\hline
\end{tabular}

Table 5: Agreement detected between positive H. pylori-DNA (PCR) in BMA and severe ITP. 
Citation: Faranoush M, Noorbakhsh S, Mehrvar A, Tabatabae Z (2013) Role of H. pylori Infection (Serology, PCR) in Chronic Idiopathic Thrombocytopenic Purpura in an Endemic Country: A Case Control Study, Tehran, IRAN. J AIDS Clin Res 4: 209. doi:10.4172/2155-6113.1000209

\begin{tabular}{|l|l|l|lc|}
\hline \multirow{2}{*}{ Total } & \multicolumn{3}{|l|}{ Severity } & \\
\cline { 1 - 3 } & 2.00 & 1.00 & & \\
\hline 3 & 0 & 3 & 1.00 & PCR HP \\
\hline 48 & 16 & 32 & 2.00 & YLO \\
\hline 51 & 16 & 35 & Total & \\
\hline
\end{tabular}

Table 6: PCR- HP Severity Cross tabulation Count

\section{References}

1. George JN (2009) Definition, diagnosis and treatment of immune thrombocytopenic purpura. Haematologica 94: 759-762.

2. Scandellari R, Allemand E, Vettore S, Plebani M, Randi ML, Fabris F (2009) Platelet response to Helicobacter pylori eradication therapy in adult chronic idiopathic thrombocytopenic purpura seems to be related to the presence of anticytotoxin-associated gene A antibodies. Blood Coagul Fibrinolysis 20: 108113

3. Pellicano R, Franceschi F, Saracco G, Fagoonee S, Roccarina D, et al. (2009) Helicobacters and extragastric diseases. Helicobacter 14 Suppl 1: 58-68.

4. Arnold DM, Bernotas A, Nazi I, Stasi R, Kuwana M, et al. (2009) Platelet count response to $\mathrm{H}$. pylori treatment in patients with immune thrombocytopenic purpura with and without $\mathrm{H}$. pylori infection: a systematic review. Haematologica 94: 850-856.

5. Figura N, Franceschi F, Santucci A, Bernardini G, Gasbarrini G, et al. (2010) Extragastric manifestations of Helicobacter pylori infection. Helicobacter 15 Suppl 1: 60-68.

6. Andersen LP (2007) Colonization and infection by Helicobacter pylori in humans. Helicobacter 12 Suppl 2: 12-15.

7. Ferrara M, Capozzi L, Russo R (2009) Effect of Helicobacter pylori eradication on platelet count in children with chronic idiopathic thrombocytopenic purpura. Hematology 14: 282-285.

8. Hayashi H, Okuda M, Aoyagi N, Yoshiyama M, Miyashiro E, et al. (2005) Helicobacter pylori infection in children with chronic idiopathic thrombocytopenic purpura. Pediatr Int 47: 292-295.

9. Amedei A, Cappon A, Codolo G, Cabrelle A, Polenghi A, et al. (2006) The neutrophil-activating protein of Helicobacter pylori promotes Th1 immune responses. J Clin Invest 116: 1092-1101.

10. Taylor JM, Ziman ME, Huff JL, Moroski NM, Vajdy M, et al. (2006) Helicobacter pylori lipopolysaccharide promotes a Th1 type immune response in immunized mice. Vaccine 24: 4987-4994.

11. Takahashi T, Yujiri T, Shinohara K, Inoue Y, Sato Y, et al. (2004) Molecular mimicry by Helicobacter pylori CagA protein may be involved in the pathogenesis of $\mathrm{H}$. pylori-associated chronic idiopathic thrombocytopenic purpura. Br J Haematol 124: 91-96.

12. Franceschi F, Christodoulides N, Kroll MH, Genta RM (2004) Helicobacte pylori and idiopathic thrombocytopenic purpura. Ann Intern Med 140: 766-767.

13. Khadem B, Imanieh MH, Gandomi B, Yeganeh F, Nik nejad N (2005) Investigation of $\mathrm{H}$ Pylori Colonization in Adenotonsillectomy Specimens by Means of Rapid Urease (CLO) Test. Iran J Med Sci 30: 138-140.

14. Saffari M, Motavalii MA, Fazeli A (2003) Immunoblot Assay in Determination of Serum Antibody Profile of Helicobacter Pylori Infection. Iran J Med Sci 28 90-93.

15. Masoodpoor N, Darakhshan, Sheikhvatan M (2008) Helicobacter pylor infection in Iranian children with recurrent abdominal pain. Trop Gastroenterol 29: 221-223.

16. Farhadi M, Noorbakhsh S, , Ebrahimi Taj F, Tabatabaei A, Javahertrash $\mathrm{N}$ et al. (2011) Unusual infections in resected adenoid of children: PCR for $C$. pneumonia, M.pneumonia, H.pylori. Eas J Med 16: 32

17. Baghaei K, Shokrzadeh L, Jafari F, Dabiri H, Yamaoka Y, et al. (2009) Determination of Helicobacter pylori virulence by analysis of the cag pathogenicity island isolated from Iranian patients. Dig Liver Dis 41: 634-638.

18. Nouraie M, Latifi-Navid S, Rezvan H, Radmard AR, Maghsudlu M, et al. (2009) Childhood hygienic practice and family education status determine the prevalence of Helicobacter pylori infection in Iran. Helicobacter 14: 40-46.

19. Rostami N, Keshtkar-Jahromi M, Rahnavardi M, Keshtkar-Jahromi M, Esfahan FS (2008) Effect of eradication of Helicobacter pylori on platelet recovery in patients with chronic idiopathic thrombocytopenic purpura: a controlled trial Am J Hematol 83: 376-381.

20. Farhadi M, Noorbakhsh S, Tabatabaei A (2011) H. pylori-DNA in nasal polyp tissues: A case-control study". IJP 